\title{
Comparing Alternative Approaches for Mobile Content Delivery in Information-Centric Networking
}

\author{
Feixiong Zhang \\ WINLAB, Rutgers University, North Brunswick, NJ, USA \\ feixiong@winlab.rutgers.edu
}

\begin{abstract}
The information-centric networking (ICN) concept has been investigated to support mobile content delivery, in which content is distributed and requested directly through their names. Pervasive in-network caching is normally enabled in ICN to further facilitate mobile content delivery. Several different architectures have been proposed to realize ICN. In this work, we present their common features and then compare different approaches for mobile content delivery in these architectures. Such comparison would greatly deepen our understanding of different design choices and help with future ICN research.
\end{abstract}

\section{INTRODUCTION}

The tremendous growth of mobile data traffic [1] has attracted research on mobile content delivery techniques. The idea of information-centric networking (ICN) [2] has been intensively discussed in these years to address mobile content delivery from a clean-slate network architecture perspective. ICN is built upon a name-based service model: a network entity can directly distribute or request content through content name at the network layer. Such model considers content as first-class network object, which is completely different from the point-to-point communication model between two end hosts in traditional Internet. Moreover, ICN normally proposes pervasive in-network caching in which each router can cache content and satisfy subsequent requests.

Several different ICN architectures are proposed, e.g., MobilityFirst (MF) [3], named data networking (NDN) [4], and XIA [5]. While sharing same ICN rationale, they differ from each other in how they realize name-based service, and how cached content is appropriately utilized by content requests. Some existing comparison of ICN [6] has investigated general aspects including routing table size, update overhead, and infrastructure requirements. In this work, we would conduct an in-depth comparison study of alternate approaches for mobile content delivery in such ICN architectures, in particular, MF and NDN. We discuss their common features and analyze the cost and benefit of different approaches.

\section{Shared Features IN ICN}

ICN architectures generally share the following features.

Name-based service: ICN designs are centered around a name-based service, i.e., they identify a network object by its unique name/identifier instead of a network address. Thus, the communication with any network object (e.g. mobile endpoint) appears to be no different than with a fixed endpoint, resulting 978-1-4799-8461-9/15/\$31.00 2015 IEEE in a location-independent communication paradigm. An abstraction of the form get(content_name) is directly supported by such name-based service and utilized to request content by the client.

In-network caching: ICN architectures support pervasive in-network caching in which the router has a cache store (CS) component in additional to the routing table. The ICN router can thus cache pass-by data and store the content for the longest useful time in order to satisfy subsequent requests locally in its cache. This is radically different from IP networks where routers transfer and discard IP packets in the shortest time possible. The ICN network thus becomes a network of caches, instead of a simple interconnection of nodes.

Caching policy: The cache store (CS) at ICN supports different caching algorithms and replacement policies. The cache algorithm determines whether to cache a data. Some typical algorithms [7] include leave copy everywhere (LCE), leave copy down (LCD), move copy down (MCD), and probabilistic caching. In addition, LRU replacement policy is normally used to determine which cached data to replace.

\section{QUALITATIVE COMPARISON OF ICN}

ICN enables the client to request content directly by name, and the network would then retrieve the content to the client by itself. Realizing such name-based service is non-trivial, especially in the presence of mobility. In this section, we discuss about how different ICN architectures realize the name-based service, and how content cache could be optimally explored by requests.

\section{A. Name-based Service and Mobility Management}

To realize named-based service, we categorize the ICN proposals into the following three approaches: indirection (Figure 1(a)), dynamic name resolution (Figure 1(b)), and name-based routing (Figure 1(c)).

The indirection approach (e.g., Home Agent and Foreign Agent in mobile IP [8]) normally uses a centralized server external to the network layer to provide the mobility bookkeeping. During content delivery, the data is first transferred to the home address, and then redirected to foreign address by querying the indirection agent if the client moves. As seen, the approach can potentially cause triangle routing which incurs unnecessary large delay for content delivery, and the indirection agent could be a single point of failure. Therefore, the approach is not an efficient and scalable mechanism for mobile content delivery in ICN. 


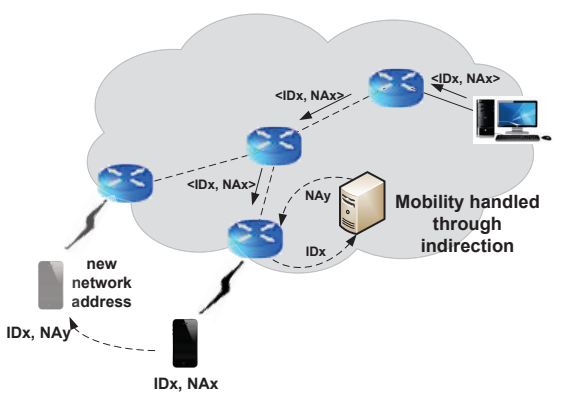

(a) Re-route through indirection

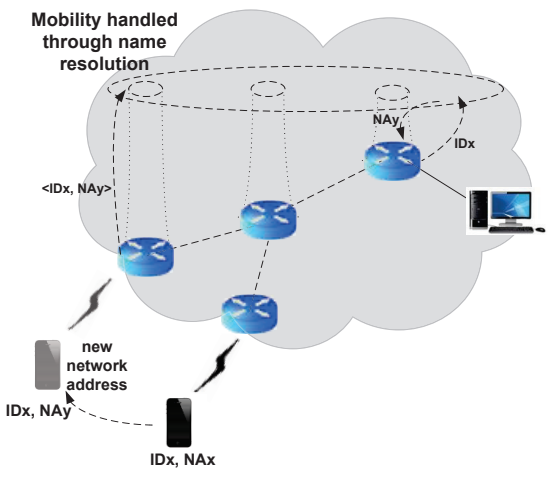

(b) Mapping through resolution service

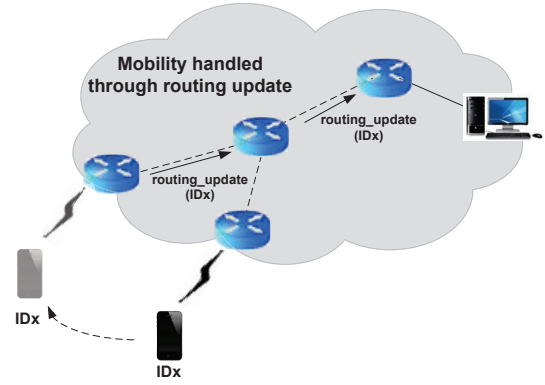

(c) Name-based routing approach

Fig. 1: Alternative approaches for handling mobility and realizing name-based service

The name resolution approach (e.g., GNRS in MobilityFirst architecture [3]) utilizes a global distributed network service to resolve a destination's name to its current address. The name service manages the object name to network address mappings and provides fast responses to mapping requests and changes, such as inserts, lookups, and updates. Such name service can be implemented in different ways: like DMAP [9], Auspice [10] and DONA [11]. Enabling mobile content delivery through dynamic name resolution where both end-hosts and routers can query [9], [10], is very efficient as it only introduces a round-trip lookup latency without significantly lengthening the data path.

A name-based routing approach (e.g. NDN) includes object name into the router's forwarding table and forwards packets directly based on the name, without using networklevel addresses at all. This approach is intellectually ideal and conceptually simple for content delivery: a client simply issues requests with content name, and the network could forward the request without any resolution; when a client moves to a new network location during content delivery, the client would reissue a content request and an intermediate cache copy might serve the request. However, it may become a challenge for mobile content delivery when the client frequently transits from one network to another. Moreover, when a content object moves, the network's routing plane needs to be updated accordingly, the propagation of routing updates usually causes substantial overhead and delays for a large network.

\section{B. Content Caching and Request Forwarding}

ICN architectures enable in-network caching and support different caching algorithms. However, they differ from each other in whether and how a content cache copy could be appropriately propagated to the network routing plane and explored by content requests. Under name-based routing approach like NDN, advertising cache information into the routing plane becomes impossible because of potential routing table explosion. In NDN, a content request is forwarded towards the original content repository, while cache copies are opportunistically explored for on-path nodes. Although authors in [7] propose scoped flooding scheme to explore off-path cache for content request and thus approximate nearest replica routing, the method can potentially cause lots of extra traffic and doesn't work for inter-domain scenarios. In MF, content cache could be advertised to GNRS, thus by querying the GNRS, a nearest replica could always be found for a content request. Therefore, while NDN avoids request resolution delay, it potentially ends up with higher data path stretch; on the other hand, MF achieves optimal content replica routing at a cost of lookup/resolution latency.

\section{Quantitative Evaluation And Future Work}

We are currently implementing a discrete-event simulator for a quantitative evaluation of alternative approaches for mobile content delivery in ICN architectures. We would like to quantify the benefit of adopting pervasive caching over simpler edge caching, and supporting optimal nearest replica routing over simply forwarding towards repository. We plan to investigate both intra-domain and inter-domain network scenarios, and look at metrics including traffic load of request and data, path stretch of request and data, etc.

\section{REFERENCES}

[1] Cisco: Global Mobile Data Traffic Forecast Update, 2014-2019.

[2] B. Ahlgren, C. Dannewitz, C. Imbrenda, D. Kutscher, and B. Ohlman, "A survey of information-centric networking," Communications Magazine, IEEE, vol. 50, no. 7, pp. 26-36, 2012.

[3] MobilityFirst project, http://mobilityfirst.winlab.rutgers.edu/.

[4] Named data networking project, http://www.named-data.net/.

[5] D. Han, A. Anand, F. R. Dogar et al., "Xia: Efficient support for evolvable internetworking." in USENIX NSDI, 2012.

[6] A. Baid, T. Vu, and D. Raychaudhuri, "Comparing alternative approaches for networking of named objects in the future internet," in Computer Communications Workshops (INFOCOM WKSHPS), 2012 IEEE Conference on. IEEE, 2012, pp. 298-303.

[7] G. Rossini and D. Rossi, "Coupling caching and forwarding: Benefits, analysis, and implementation," in Proceedings of the 1st international conference on Information-centric networking. ACM, 2014, pp. 127136.

[8] S. Gundavelli, K. Leung, V. Devarapalli, K. Chowdhury, and B. Patil, "Proxy mobile ipv6," RFC 5213, August, Tech. Rep., 2008.

[9] T. Vu et al., "Dmap: A shared hosting scheme for dynamic identifier to locator mappings in the global internet," in IEEE ICDCS, 2012.

[10] X. Tie, A. Sharma, and A. Venkataramani, "A global name service for a highly mobile internet," Technical report, UMASS, Tech. Rep., 2013.

[11] T. Koponen, M. Chawla, B.-G. Chun, A. Ermolinskiy et al., "A dataoriented (and beyond) network architecture," ACM SIGCOMM Computer Communication Review, vol. 37, no. 4, pp. 181-192, 2007. 